\title{
Accumulation of high magnitude acceleration events predicts cerebrovascular reactivity changes in female high school soccer athletes
}

Diana O. Svaldi, 1,2四

Phone (317) 417 - 9347

Emaildianaotero7@gmail.com

Chetas Joshi, 3

Emily C. McCuen,

Jacob P. Music, 4

Robert Hannemann, 1,5,6,7

Larry J. Leverenz, 8

Eric A. Nauman, 1,4

Thomas M. Talavage, 1,3

1 Weldon School of Biomedical Engineering, Purdue University, West

Lafayette, IN, 4907 USA

2 Department of Neurology, Indiana University School of

Medicine, Indianapolis, IN, 46202 USA

3 School of Electrical and Computer Engineering, Purdue University, West

Lafayette, IN, 47907 USA

4 School of Mechanical Engineering, Purdue University, West 
Lafayette, IN, 47907 USA

5 School of Chemical Engineering, Purdue University, West

Lafayette, IN, 47907 USA

6 Department of Child Psychology, Purdue University, West Lafayette, IN, 47907 USA

7 Department of Pediatrics, Indiana University School of Medicine, Indianapolis, IN, 46202 USA

8 Department of Health and Kinesiology, Purdue University, West Lafayette, IN, 47907 USA

\section{Abstract}

Mitigating the effects of repetitive exposure to head trauma has become a major concern for the general population, given the growing body of evidence that even asymptomatic exposure to head accelerations is linked with increased risk for negative life outcomes and that risk increases as exposure is prolonged over many years. Among women's sports, soccer currently exhibits the highest growth in participation and reports the largest number of mild traumatic brain injuries annually, making female soccer athletes a relevant population in assessing the effects of repetitive exposure to head trauma. Cerebrovascular biomarkers may be useful in assessing the effects of repetitive head trauma, as these are thought to contribute directly to neurocognitive symptoms associated with mild traumatic brain injury. Here we use fMRI paired with a hypercapnic breath hold task along with monitoring of head acceleration events, to assess the relationship between cerebrovascular brain changes and exposure to repetitive head trauma over a season of play in female high school soccer athletes. We identified longitudinal changes in cerebrovascular reactivity that were significantly associated with prolonged accumulation to high magnitude ( $>75$ th percentile) head acceleration events. Findings argue for active monitoring of athletes during periods of exposure to head acceleration events, illustrate the 
importance of collecting baseline (i.e., pre-exposure) measurements, and suggest modeling as a means of guiding policy to mitigate the effects of repetitive head trauma.

AQ1

\section{Keywords}

Cerebrovascular Reactivity

Functional magnetic resonance imaging

Concussion

Mild traumatic brain injury

Subconcussive injury

Soccer

\section{Electronic supplementary material}

The online version of this article ( https://doi.org/10.1007/s11682-018-9983-0 ) contains supplementary material, which is available to authorized users.

\section{Introduction}

There is a growing body of evidence that a history of head trauma is associated with increased risk for later life dysfunction even in the absence of physician diagnosed mild traumatic brain injury (mTBI). Consequently the mitigation of repetitive exposure to subconcussive events - i.e., head acceleration events (HAEs) involving direct impact to or whiplash of the head, but for which there is no associated diagnosis of "concussion"- -has become a major concern for the general population (Talavage et al. 2015; Bailes et al. 2013). Accumulation of subconcussive HAEs has been associated with elevated risk of negative cognitive outcomes later in life (Montenigro et al. 2017; Stamm et al. 2015). Critically, HAEs not associated with a classification of "concussed" have been demonstrated to increase the incidence of chronic traumatic encephalopathy (CTE) in an animal model (Tagge et al. 2018). From the perspective of severity, late life cognitive and structural abnormalities have been shown to be exacerbated by the onset of this cumulative exposure before the age of twelve in retired NFL athletes exhibiting 
cognitive, behavioral, and mood symptoms (Stamm et al. 2015; Schultz et al. 2017). Though the sample sizes for these studies have been small and limited to symptomatic retired NFL athletes, significant differences in long term outcomes between athletes who began experiencing subconcussive HAE exposure before and after the age of 12 indicate the need to assess the effects of exposure to such HAEs at developmental ages. Even in the case of CTE-wherein $16 \%$ of pathologically confirmed cases are in individuals with no history of diagnosed mTBI-it is years of exposure, rather than number of previous diagnosed mTBIs, that best relates to severity (Stein et al. 2015).

Many neuroimaging biomarkers have proven to be sensitive to brain changes occurring in asymptomatic athletes during, and subsequent to, periods of exposure to repetitive head trauma (Koerte et al. 2015). These biomarkers include taskbased FMRI (Talavage et al. 2014; Shenk et al. 2015), diffusion-weighted imaging measures (Bazarian et al. 2014; Davenport et al. 2014; Chun et al. 2015; Bahrami et al. 2016; Davenport et al. 2016; McAllister et al. 2014; Lipton et al. 2013), MR spectroscopic measures of brain metabolism (Poole et al. 2014), resting-state functional connectivity as assessed using FMRI (Johnson et al. 2014; Abbas et al. 2015a; Abbas et al. 2015b; Slobounov et al. 2017), global measures of cerebral blood flow (Slobounov et al. 2017) and also measures associated with microhemorrages as obtained from susceptibility weighted imaging (Slobounov et al. 2017). Further, it appears that many of the deviations observed in these biomarkers are a function of the longitudinal accumulation of HAEs during the season (Robinson et al. 2015; Breedlove et al. 2012; Poole et al. 2015; Talavage et al. 2014; Talavage et al. 2015).

In addition to the aforementioned biomarkers, whole-brain cerebrovascular reactivity (CVR) to $\mathrm{CO}_{2}$, as measured using functional magnetic resonance imaging (FMRI) paired with a hypercapnic task, has proven to be sensitive to brain alterations following exposure to repetitive head acceleration in asymptomatic athletes (Svaldi et al. 2017; Svaldi et al. 2015). CVR is a compensatory mechanism whereby blood vessels react to regulate cerebral blood flow (CBF) in response to hypercapnic and hypocapnic stimuli (Lewis et al. 2014). Measures of CVR have previously been documented to be sensitive to symptomatic TBI (Len et al. 2013; 
Chan et al. 2015; Ellis et al. 2016; Mutch et al. 2014; Mutch et al. 2016), and impaired CVR is thought to be a key contributor in neurocognitive symptoms associated with mTBI (Giza and Hovda 2001; Barkhoudarian et al. 2011), lending credence to its application in the asymptomatic case.

Female collision sport athletes are known to exhibit higher rates of mild (Poole et al. 2015; Poole et al. 2014; Abbas et al. 2015b; Svaldi et al. 2015) TBI (e.g., concussion) and increased symptom severity relative to male athletes (Broshek et al. 2005) indicating the need to study the cumulative effects of exposure to repetitive head trauma in female populations. Of women's sports, soccer reports the largest growth in participation (Morris 2015), highest numbers of mTBI annually (Marar et al. 2012; Delaney et al. 2002). Additionally, heading in soccer has been associated with adverse neurocognitive symptoms (Stewart et al. 2017; Lipton et al. 2013) and structural white matter changes (Lipton et al. 2013), making women's soccer a relevant population in the study of effects associated with repetitive head trauma in females.

Previous evaluation of CVR in female high school soccer athletes found global decreases in this measure, relative to baseline, for those athletes in the top half of subconcussive HAE accumulation over a season of play (Svaldi et al. 2017). This finding was consistent with prior evaluation in male high school football athletes (Svaldi et al. 2015), and suggests that a relationship should exist between the longitudinal accumulation of HAEs and the growth of the deviation of CVR from baseline. This study was undertaken to model the relationship between (subconcussive) HAE accumulation and CVR changes in a cohort of female high school soccer athletes. Previous work indicated that a significant negative relationship should exist between growing accumulation of HAEs and CVR changes, relative to a pre-participation baseline-i.e., greater decreases in CVR are expected with increased accumulation of subconcussive events. Evidence presented here of a relationship between daily accumulation of high magnitude subconcussive HAEs and changes in CVR argues for more active monitoring of athlete exposure to HAEs, and suggests the potential for use of predictive modeling to guide proactive mitigation of exposure in athletes who are most prone to HAE accumulation. 


\section{Methods}

Participants 21 high school aged female soccer athletes (ages 15-18; mean $15.9 \pm 1.0)$ participated voluntarily in this research. No included participant was diagnosed with a concussion or other form of (mild) traumatic brain injury during the period of study. Six of 21 athletes had a previously diagnosed concussion (1 previous $=4$ players; 2 previous $=1$ player; 3 previous $=1$ player $)$. Soccer athlete data were collected over two seasons of play, with six of the 21 athletes participating in imaging as part of the study for both seasons. Our previous work (Svaldi et al. 2017) reported that CVR in these six athletes returned to preparticipation levels by one year following their initial Pre scan (see below). Therefore, to investigate the deviation of CVR as a function of head acceleration event (HAE) exposure, imaging data from consecutive seasons were treated as separate observations, yielding a total of 27 athlete-season observations.

Imaging schedule All athletes underwent multiple MR imaging sessions during a single competition season, including the period of pre-season conditioning/training. Soccer athletes were imaged once before the onset of the current season's collision activities (Pre), once each during the first half (approximately 5 weeks) of the contact season (In1) and the second half (In2), and once at an interval of 1-2 months after the end of the competition season (Post). Pre scans took place 8 months following the previous HS season and approximately 2-3 months following the previous club/travel season. Note that these athletes were also imaged 3-4 months after the end of the season; however, as survey data revealed that most athletes were now participating in club/travel soccer in the absence of monitoring of head acceleration events, data from this session were not included in this analysis.

Imaging protocols All MR imaging was conducted at the Purdue University MRI Facility (West Lafayette, IN), using a 3 T General Electric Signa HDx (Waukesha, WI) with a 16-channel brain array (Nova Medical; Wilmington, MA). CVR was measured using a hypercapnic breath hold challenge, as described in (Svaldi et al. 2017), which has been shown to produce good repeatability in the human brain (Kastrup et al. 2001; Bright and Murphy 2013; Lipp et al. 2015). For 
each imaging session, a single blocked breath-hold FMRI run (4 breath holds, 20s duration, separated by paced breathing, hold on the exhale) was acquired in each session using a gradient-echo echo planar sequence $(\mathrm{TR} / \mathrm{TE}=1500 / 26 \mathrm{msec}$; $20 \mathrm{~cm}$ FOV; $64 \times 64$ matrix; 34 slices; $3.8 \mathrm{~mm}$ thickness; 117 volumes). PsychoPy (Peirce 2007) was used to cue the task with instructions presented via a NordicNeuroLab fiber optic visual system. A respiration belt was used to monitor task compliance. For registration purposes across sessions and subjects, a T1weighted anatomical scan was acquired using a 3D spoiled gradient echo sequence (TR/TE $5.758 \mathrm{~ms} / 2.032 \mathrm{~ms}$, flip angle $=73^{\circ}, 1 \mathrm{~mm}$ isotropic resolution). Due to failure in pre-processing of either the fMRI data or the respiratory data, certain subject-sessions had to be excluded from final analyses. After pre-processing, the final sample sizes at each session were $\mathrm{N}_{\text {Pre }}=27, \mathrm{~N}_{\text {In } 1}=27, \mathrm{~N}_{\text {In } 2}=26, \mathrm{~N}_{\text {Post }}=26$.

\section{Head acceleration event (HAE) analysis HAEs experienced by soccer} athletes were monitored using an xPatch (X2 Biosystems; Seattle, WA) throughout the entire season of participation, including games and practices-cf. McCuen et al. (2015). Sensors were placed behind the right ear with an adhesive patch (Cummiskey et al. 2017). The sensors monitored (1 kHz sampling rate) three axes of translational acceleration and three axes of angular velocity. Acceleration events were recorded when the net translational acceleration exceeded a $10 \mathrm{~g}$ threshold. Features within the software provided peak translational acceleration (PTA) measurements for each detected event. All HAEs recorded during games and practices were included regardless of the X2's classification of valid versus invalid events. Given no subjects were diagnosed with a concussion during the course of this study, all recorded HAEs are considered hereafter to be "subconcussive" HAEs.

As our previous work suggested accumulation of subconcussive trauma with each experienced event (Svaldi et al. 2017), the cumulative PTA (CPTA) was computed at each imaging session for evaluation of predictive relationships between aggregate mechanical loading and changes in CVR. To test the contribution of various severities of HAEs to changes in CVR, a minimum PTA threshold was defined and used to constrain the subset of HAEs that were included in the CPTA calculation. Specifically, CPTA values were computed for each athlete, at each 
session, using four minimum PTA thresholds (Th): $20 \mathrm{~g}, 30 \mathrm{~g}, 50 \mathrm{~g}$, and $70 \mathrm{~g}$ (approximately corresponding to the 25th, 50th, 75th, and 90th percentile for all HAEs recorded over the two seasons). Though commonly recorded in our cohort, accelerations below $20 \mathrm{~g}$ were discarded from analyses as they were typically not associated with direct impacts to the head, but rather with events such as stops, cuts, or forceful kicks that are unlikely to result in adverse neurophysiological changes (McCuen et al. 2015). While there is concern in the community regarding reports that the xPatch and other telemetry devices frequently indicate acceleration events that are not subsequently confirmed by video to be true impacts to the head, we focus here on all head accelerations events exceeding $20 \mathrm{~g}$-falling within range of typical impacts to the head for our cohort (McCuen et al. 2015) - given evidence that head acceleration events in the absence of impacts may still be deleterious to the brain (Meaney and Smith 2011).

For the $i$-th soccer athlete at the $j$-th follow-up session, the (to-date) threshold dependent cumulative PTA (CPTA $T h, j, i$; see Eq.1) was calculated by summing the PTAs of the $N_{j}$ HAEs experienced prior to that session by the given athlete that exceeded the threshold:

$$
C P T A_{T h, j, i}=\sum_{k=1}^{N_{j}} P T A_{i, k} \bullet u\left(P T A_{i, k}-T h\right)
$$

where

$$
u(x)= \begin{cases}1 & \text { if } x>0 \\ 0 & \text { if } x \leq 0\end{cases}
$$

Because imaging sessions (In1, In2, Post) were conducted over spans of five weeks and CPTA was only correlated to imaging date for the In 1 follow-up session, CPTA was normalized (nCPTA) by the number of days into the season at which the $j$-th imaging session took place for the $i$-th athlete $\left(D_{j, i}\right)$ :

$$
n C P T A_{T h, j, i}=\frac{C P T A_{T h, j, i}}{D_{j, i}}
$$


Note that for the Post session, $D_{j, i}$ was taken as the total number of days over which the competitive season had taken place.

To characterize the variability in HAE accrual at a given session and the importance of normalizing $C P T A_{T h, j, i}$ by $D_{j, i}$, athletes were divided into High, Medium, and Low loading groups defined as the top, middle, and bottom thirds of the $C P T A_{T h, j, i}$ distribution of all athlete observations pooled, over both seasons ( $n$ $=27)$.

Analysis of cerebrovascular reactivity The same processing and regression pipeline as described in Svaldi et al. (2017) was used here to process the FMRI data to obtain a final optimized measure of CVR. However, for this work the four breath-hold periods were modeled as a single duration modulated regressor rather than three separate regressors of the same class. In our previous work (Svaldi et al. 2017) soccer athletes exhibited primarily whole brain CVR changes relative to baseline. Therefore, here we used the $\beta$-Weight (regression coefficient) from the latency optimized regression of the whole gray matter (GM) FMRI time-series against the breath hold regressor as the final quantification of CVR. The fractional change in $\beta$-Weight from Pre- $-\Delta \beta_{j, i}$-was calculated at each follow-up session for each subject, and used to model relationships with HAE.

To assess distributions of $\Delta \beta_{j, i}$ at each follow-up session, 10,000 bootstrapped random samples (with replacement) of $\Delta \beta_{j, i}$ were generated and the mean of each sample $-\Delta \beta_{j}^{*}$ - was calculated. The mean of the distribution of 10,000 values of $\Delta \beta_{j}^{*}-\widehat{\Delta \beta}_{j}$ - was used as the final estimate of mean fractional CVR change from baseline. A non-parametric $95 \%$ confidence interval around $\widehat{\Delta \beta}_{j}$ was used to assess whether the distribution (i.e., the change in CVR for that session) was significantly different from zero indicating an effect of session on CVR, relative to Pre. Additionally, Friedman's non-parametric ANOVA was conducted using 25 (of the 27) athletes who had $\Delta \beta_{j, i}$ measurements at all sessions, to test for an effect of follow-up session on $\Delta \beta_{j, i}$. As no effect of session was found, pairwise follow-up tests were not conducted. 
Effects of cumulative exposure on CVR changes To assess the effect of nCPTA on CVR changes over a season, a linear fit (Original Least Squares; see Eq. 3 ) was used to model the relationship between $n C P T A_{T h, j, i}$ and $\Delta \beta_{j, i}$ for each value of $T h$. The significance of each model was assessed using an F-test $(\alpha=$ $0.05)$.

$$
\widehat{\Delta \beta}_{T h, j, i}=\widehat{x}_{0, T h, j}+\widehat{x}_{1, T h, j} * n C P T A_{T h, j, i}
$$

To robustly assess coefficient distributions given the limited sample size, 10,000 iterations of bootstrapped random sampling of the observations (with replacement) were conducted. At each iteration, the final model was fit to yield 10,000 estimates of the regression coefficients $\left(x_{0, T h, j}^{*}, x_{1, T h, j}^{*}\right)$ as well as the goodness-of-fit, $R_{T h, j}^{2 *}$. The means of the distributions generated for each coefficient $\left(\hat{x}_{0, T h, j}, \hat{x}_{1, T h, j}\right)$ and the goodness-of-fit ( $\left.\widehat{R}^{2} T h, j\right)$ were used as the final estimates for the regression. A non-parametric, percentile based $95 \%$ confidence interval (CI) was also calculated for each of these three estimates. $n C P T A_{T h, j, i}$ was deemed to have a significant effect on $\Delta \beta_{T h, j, i}$ if zero was not included in the CI for $\hat{x}_{1, T h, j}$.

Because $n C P T A_{T h, j, i}$ exhibited a significant effect on $\widehat{\Delta \beta}_{T h, j, i}$ at the $\operatorname{In} 2$ session for $T h=50 \mathrm{~g}$ and $T h=70 \mathrm{~g}$, a post-hoc analysis was conducted to evaluate the above relationships with $T h$ on a finer scale. The bootstrap procedure described above was used to estimate model parameters for thresholds at $5 \mathrm{~g}$ intervals over the range of $T h=30-70 \mathrm{~g} . \widehat{R^{2}} T h, j$ was plotted against $T h$ to identify where the variance explained by the model was maximized.

Effect of age on CVR changes As above, a linear fit (Original Least Squares; see Eq. 4) was used to model the relationship between $a g e_{j, i}$ and $\Delta \beta_{j, i}$ at each follow-up session. The significance of each model was assessed using an F-test and follow up $t$-tests were used to assess the significance of the associated coefficients.

$$
\widehat{\Delta \beta}_{j, i}=\widehat{x}_{0, j}+\widehat{x}_{2, j} * a g e_{j, i}
$$


As no effect of age on $\widehat{\Delta \beta}_{j, i}$ was exhibited at any point in the season, no post hoc analyses were conducted.

\section{Results}

Head acceleration event monitoring Figure 1 illustrates the relationship between the $P T A_{T h, j, i}, C P T A_{T h, j, i}$, and $n C P T A_{T h, j, i}$ calculations. Fig. 1a presents $P_{20 A_{j, j, i}}$ for individual HAEs accrued over a season for two sample athletes - Low Accumulation, one representative of Low HAE accumulation (i.e., lowest third), and High Accumulation, one athlete representative of High HAE accumulation (i.e., highest third). At In 1, the Low Accumulation athlete accrued $1060 \mathrm{~g}$ over 20 days $(n C P T A=53 \mathrm{~g} /$ day $)$, increasing to $1581 \mathrm{~g}$ over 44 days $\left(n C P T A_{2}=\right.$ $36 \mathrm{~g} /$ day) at $\operatorname{In} 2$, and $4511 \mathrm{~g}$ over 82 days ( $n C P T A=55 \mathrm{~g} /$ day) by the end of the season. In contrast, the High Accumulation athlete accrued $1561 \mathrm{~g}$ over 11 days $(n C P T A=142 \mathrm{~g} /$ day $)$ by $\operatorname{In} 1,7215 \mathrm{~g}$ over 39 days $(n C P T A=185 \mathrm{~g} /$ day $)$ at $\operatorname{In} 2$, and $14,487 \mathrm{~g}$ over 82 days $(n C P T A=177 \mathrm{~g} /$ day $)$ by the end of the season.

\section{Fig. 1}

a Scatter plot of PTA versus days since the start of the season for all HAEs accrued over the season in representative athletes at High Accumulation (HA) and Low Accumulation (LA) levels. Thresholds of $30 \mathrm{~g}, 50 \mathrm{~g}$, and $70 \mathrm{~g}$ are delineated by horizontal lines. Impacts below $20 \mathrm{~g}$ are automatically discarded as per McCuen et al. (2015). b $C P T A_{20 g, j, i}$ versus days since start of the season. Values shown in the plot were calculated by summing individual PTAs associated with HAEs acquired before the given follow-up imaging session (In1, In2, Post) for all athlete observations at each session. c $n C P T A_{20 g, j, i}$ versus days since start of season for all athlete observations at each session. Values shown in the plot were calculated by normalizing CPTA values by D 
a

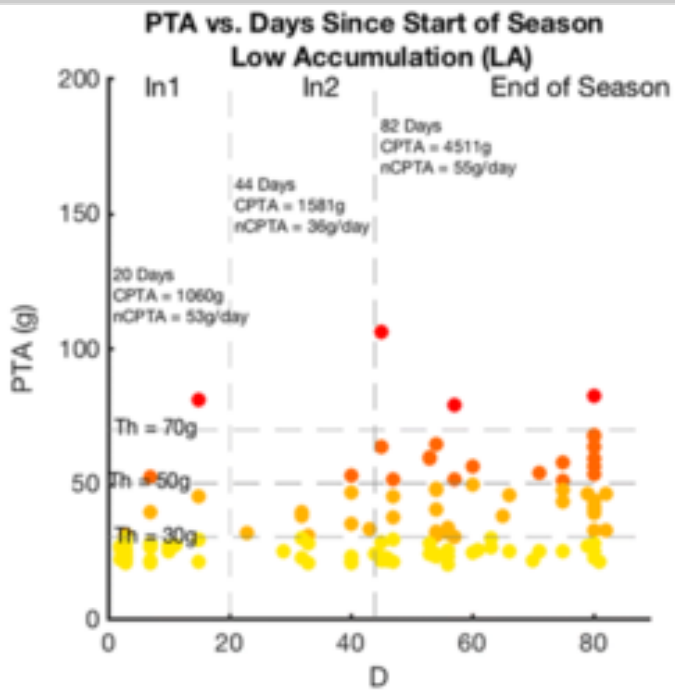

PTA vs. Days Since Start of Sea High Accumulation (HA)

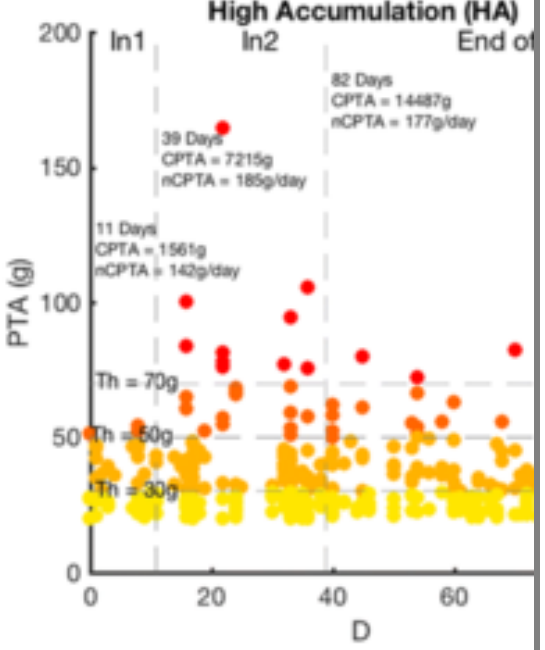

b

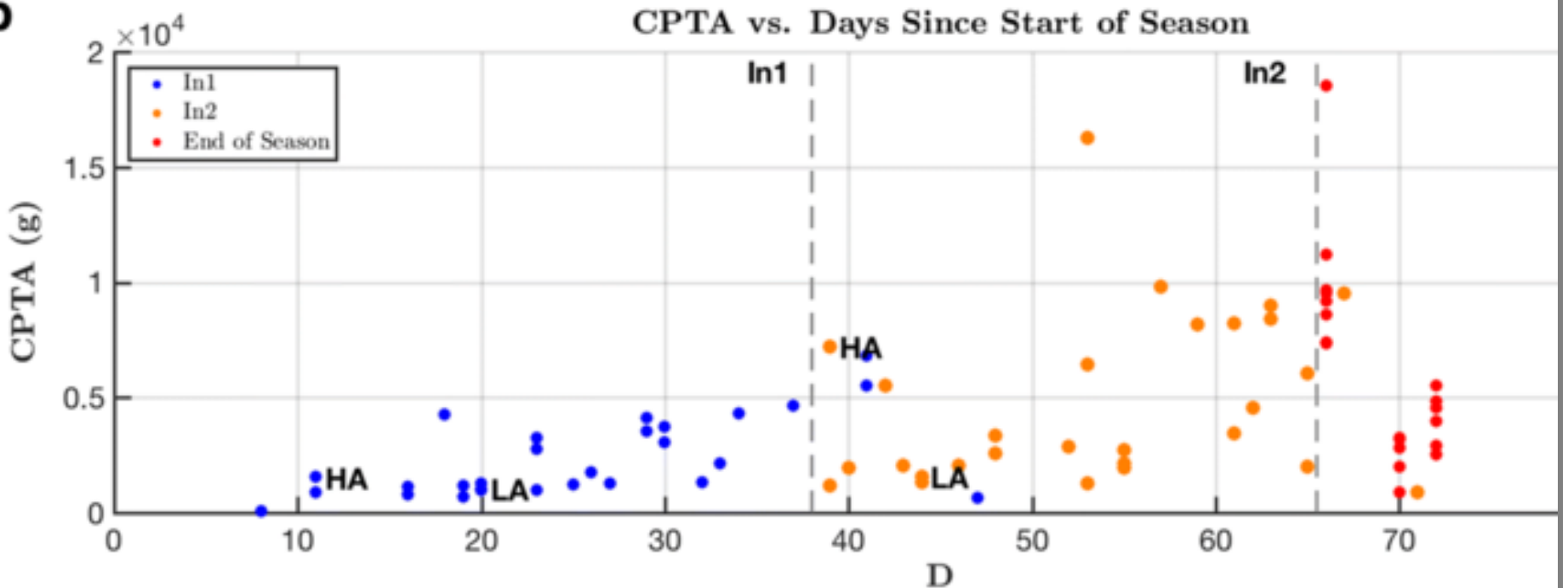

C

nCPTA vs. Days Since Start of Season

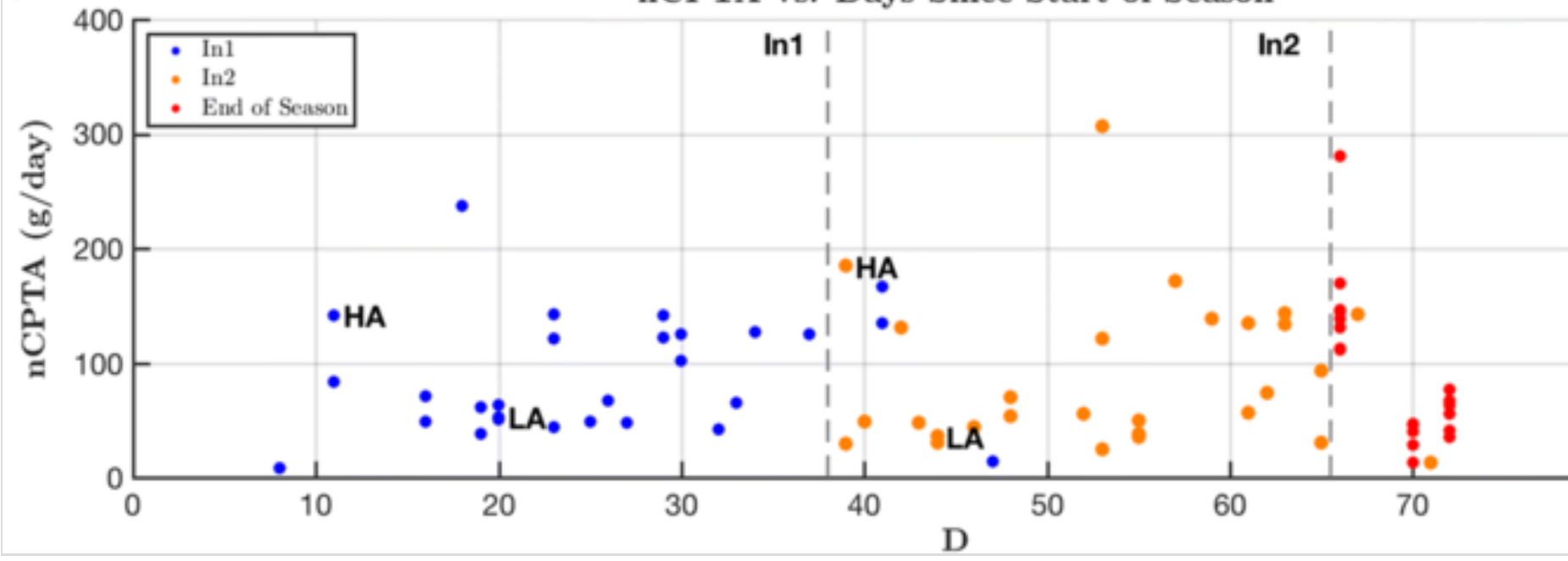


Figure $1 \mathrm{~b}$ depicts $C P T A_{T h, j, i}$ for each athlete at the time of the given imaging sessions (In 1-2) or at the end of the season (corresponding to the quantity used at the Post imaging session). CPTA accrual varies by athlete, as is exemplified by some athletes having greater CPTA at their In 1 assessment than other athletes when scanned at their In 2 assessments. Further note that High Accumulation (HA) and Low Accumulation (LA) athletes (illustrated in detail in Fig. 1a) were imaged at times that put them both in the lower half of the In 1 CPTA distribution (Fig. 1b).

Last, Fig. 1c depicts $n C P T A$ for each athlete as of their respective follow-up assessments (In 1-2) or for the total season (i.e., the value used for analysis of the Post session). Note that following normalization of CPTA by days since the start of the season, the example the High Accumulation and Low Accumulation athletes are now seen to have experienced appreciably different accrual of HAEs at the InI session. For example, the High Accumulation athlete is in the bottom half of the In1 CPTA distribution, but in the upper half of the In 1 nCPTA distribution (Fig. $1 \mathrm{c})$.

\section{CVR changes over a season}

$\widehat{\Delta \beta}_{j}$, the mean CVR fractional change from Pre, was found to be significantly decreased from zero (i.e., outside the 95\% CI; see Fig. 2) at all follow-up imaging sessions. There was no significant effect of follow-up session on $\widehat{\Delta \beta}_{j}(\alpha=0.05$, Friedman's ANOVA $)$.

\section{Fig. 2}

Distributions of estimated mean fractional change in CVR $\Delta \beta_{i, j}$ ) at each follow up session. Distributions marked with an asterisk are significantly different from zero $(\alpha=0.05$, non-parametric 95\% CI, 10,000 bootstraps). There was no significant effect of follow-up session on fractional change in CVR $\Delta \beta_{i, j}(\alpha=0.05$, Friedman's non-parametric ANOVA) 


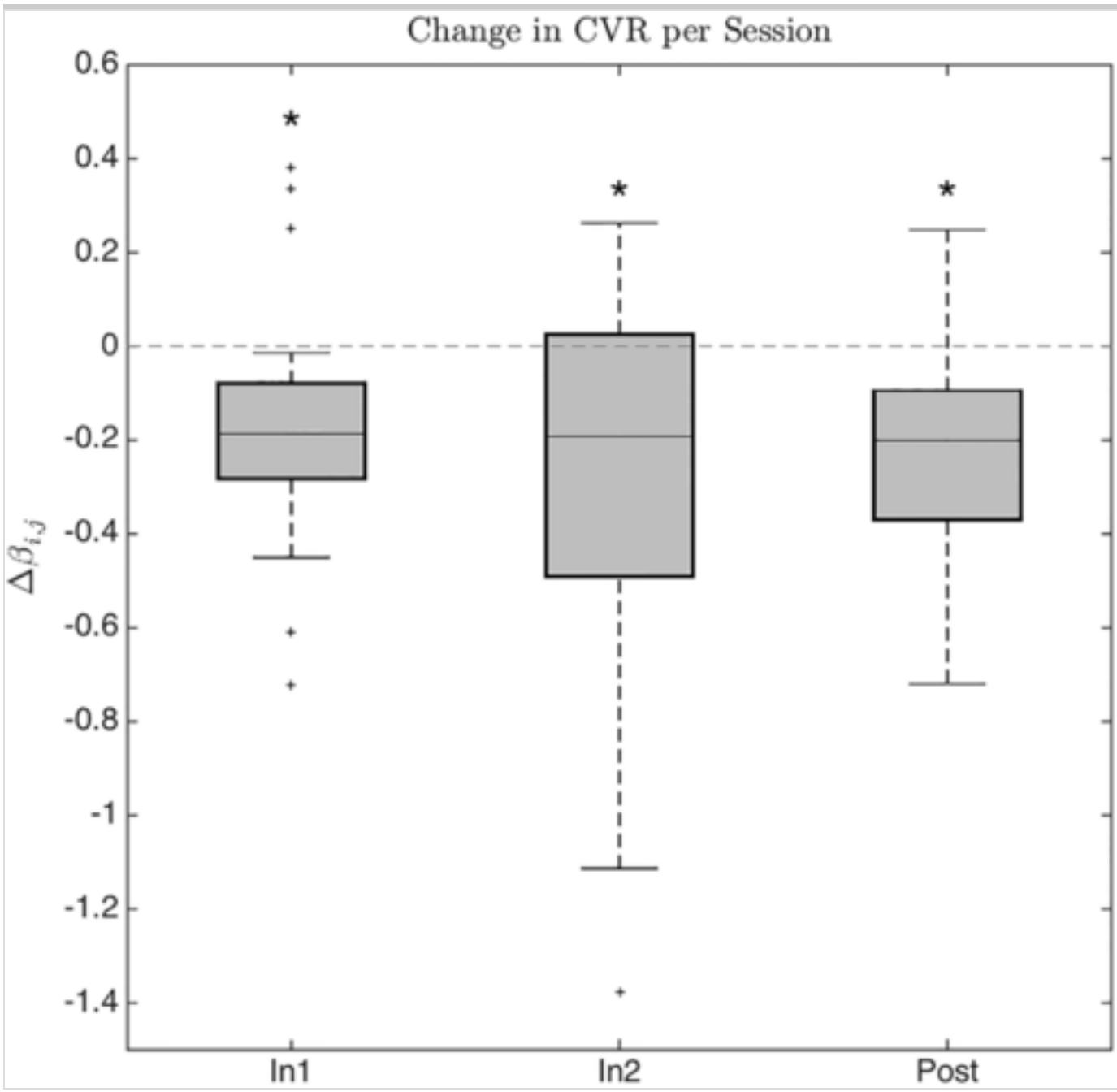

CVR changes as function of nCPTA Table 1 presents the results from fitting the model of Eq. 3, seeking to characterize the effect of $n C P T A$ on CVR. The overall fit was found to be significant only for the In 2 session, at thresholds of $50 \mathrm{~g}$ and $70 \mathrm{~g}(p<0.05$, F-test; see Table 1$)$. Following bootstrapped random sampling, the cumulative load coefficient associated with these fits was only found to be significantly different from zero for HAES above $50 \mathrm{~g}$ (non-parametric $95 \%$ CI, Fig. 3b). At this $50 \mathrm{~g}$ threshold, $n C P T A$ explained $23.3 \%$ of the variance in CVR change from Pre (Fig. 3a). No fits or coefficients were found to be statistically-significant for the In1 or Post sessions. 


\section{nCPTA}

\begin{tabular}{|c|c|c|c|c|c|}
\hline & Th (g) & $p$ & $\begin{array}{c}\widehat{x}_{0, T h, j} \\
\text { (intercept) }\end{array}$ & $\begin{array}{c}\widehat{x}_{1, T h, j} \\
(n C P T A)\end{array}$ & ${\widehat{\boldsymbol{R}^{2}}}_{T h, j}$ \\
\hline \multirow{4}{*}{ In1 } & 20 & 0.9909 & -0.155 & 0.000 & 0.035 \\
\hline & 30 & 0.9677 & -0.152 & 0.000 & 0.030 \\
\hline & 50 & 0.9284 & -0.163 & 0.000 & 0.026 \\
\hline & 70 & 0.426 & -0.201 & 0.004 & 0.058 \\
\hline \multirow{4}{*}{ In2 } & 20 & 0.325 & -0.164 & -0.002 & 0.081 \\
\hline & 30. & $\underline{0.076}$ & $=-0.095$ & -0.003 & 0.153 \\
\hline & 50 & $\underline{0.016}$ & -0.053 & -0.008 & 0.233 \\
\hline & 70 & 0.039 & -0.090 & -0.014 & 0.17 \\
\hline \multirow{4}{*}{ Post } & 20 & 0.853 & -0.199 & 0.000 & 0.047 \\
\hline & 30 & 0.937 & -0.212 & 0.000 & 0.053 \\
\hline & 50 & 0.657 & -0.231 & 0.001 & 0.067 \\
\hline & 70 & 0.268 & -0.263 & 0.004 & 0.093 \\
\hline \multicolumn{6}{|c|}{ Age } \\
\hline
\end{tabular}

$\widehat{\boldsymbol{x}}_{\mathbf{0}, j} \quad \widehat{\boldsymbol{x}}_{2, j}$

$\begin{array}{lllll}\text { Th (g) } & p & \text { (intercept) } & \text { (Age) } & \widehat{R}^{2} \\ \end{array}$

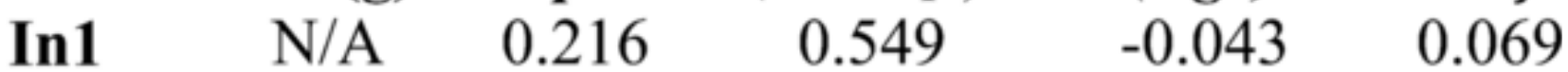

In2 $\quad \mathrm{N} / \mathrm{A} \quad 0.218 \quad 1.159 \quad-0.086 \quad 0.068$

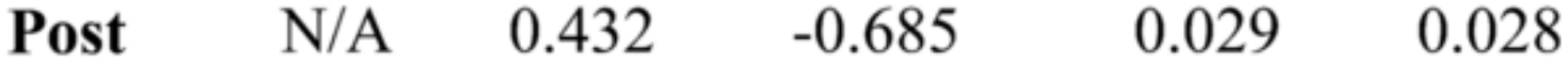




\section{Table 1}

Coefficients obtained from linear modeling of the effect of $n C P T A_{T h, j, i}$ and $A g e_{j, i}$ on $\Delta \beta_{j}$, ${ }_{i}$ at each session and for each threshold $(T h)$

Dashed red outlines delineate rows of coefficients for which the fitting was statistically significant $(\alpha=0.05$, F-test whole model)

\section{Fig. 3}

a $I n 2$ mean goodness of fit $\left(\widehat{R^{2}} T h, j\right)$ at each threshold value $(T h)$. Asterisks denote values of $T h$ for which the regression between $\widehat{\Delta \beta}_{T h, j, i}$ and $n C P T A_{T h, j, i}$ was significant (F-test, $\alpha=0.05$ ). Red data points indicate $T h$ values at which the distribution of the nCPTA coefficient $x_{1, T h, j}$ was significantly different from zero (95\% CI, non-parametric). b Linear regressions 6 $\left.\Delta \beta_{T h, i, j}=\hat{x}_{0, T h, j}+\hat{x}_{1, T h, j} * n C P T A_{T h, j, i}\right)$ and bootstrapped $\hat{x}_{1, T h, j}$ distributions significantly differing from zero $(95 \% \mathrm{CI}$, non-parametric) 


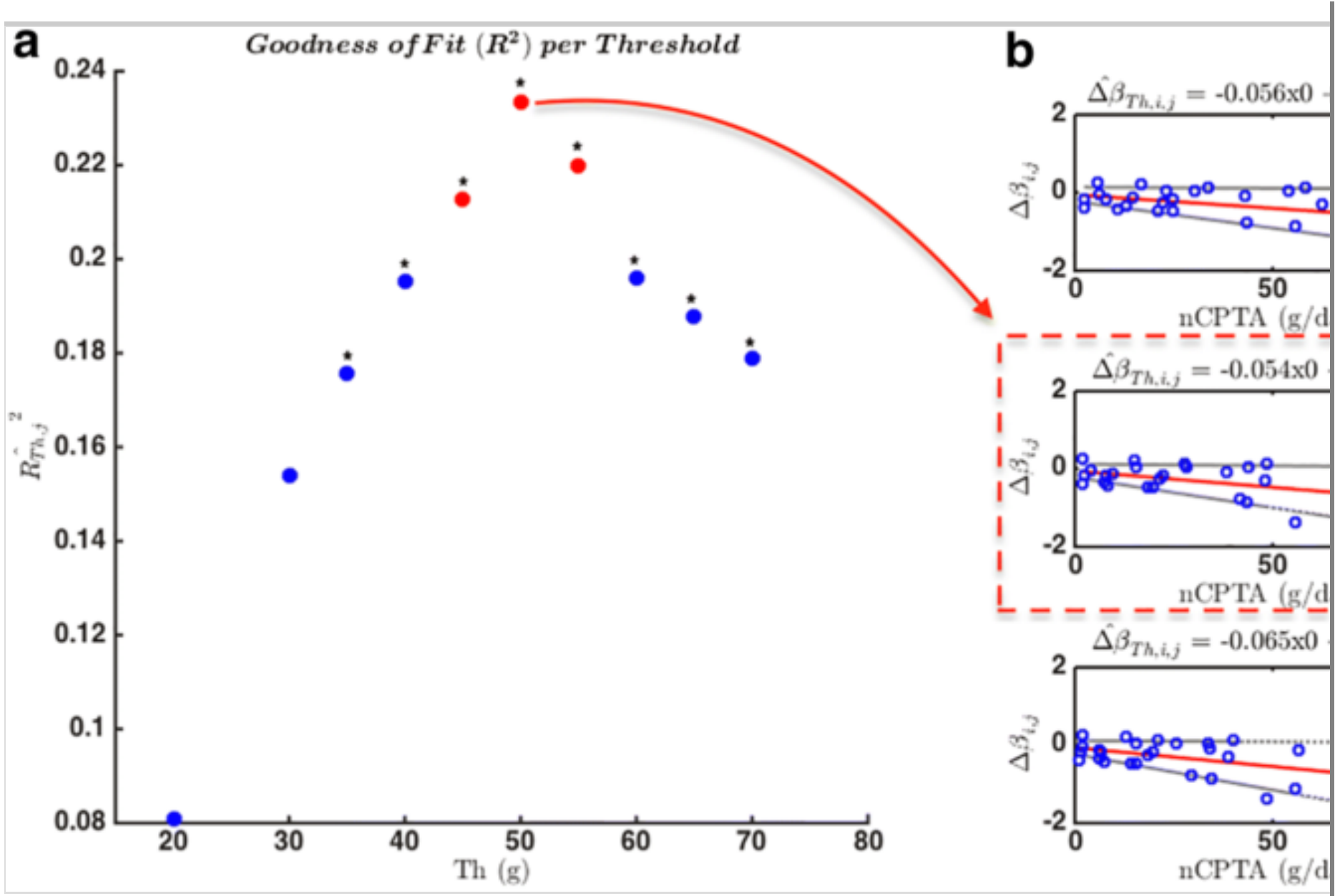

Finer threshold scale analysis CVR changes with nCPTA At In2, for all threshold values assessed in the range $T h=30 \mathrm{~g}-70 \mathrm{~g}, n C P T A$ was found to have a significant effect on CVR changes from Pre $p<0.05$, F-test; Fig. 3a), with the maximum variance explained found at a threshold of $50 \mathrm{~g}$ (Fig. 3a).

$\mathrm{AQ2}$

CVR changes as a function of age Also as presented in Table 1, there was no significant effect of age at any session on CVR changes from Pre.

\section{Discussion}

Having previously demonstrated the presence of changes in CVR in female athletes participating in high school soccer (Svaldi et al. 2017), this larger study was intended to address the critical question whether these changes were meaningfully related to the accrual of (subconcussive) HAEs over the course of a 
collision-sport season. Within-season prospective monitoring of both CVR and exposure to HAEs revealed that normalized cumulative HAE accrual measures were significantly related to CVR decreases observed during the latter half of the season, relative to pre-participation measurements. This relationship was optimized when only those impacts exceeding $50 \mathrm{~g}$ (corresponding to the 75 th percentile for all recorded impacts) were considered. No relationship was observed after an extended period (1-2 months) of rest following the end of the season, nor was a predictive relationship observed during the initial buildup of HAEs. These observations suggest the following: (1) a minimum threshold of individual HAE mechanical loading exists, below which the accrual mechanical insult does not meaningfully affect CVR regulation; and (2) there is active competition between HAE-associated accrual and natural repair and adaptation processes that counteract the accrued subconcussive trauma induced by these mechanical insults.

Comparison of findings with previous work The observed significant decreases in CVR relative to Pre (Fig. 2) are consistent with the prior literature, extending previous work through the modeling of a predictive relationship with (subconcussive) HAE exposure. Previous results on a subset of this cohort (14 athletes) found significant global decreases in CVR that were not present in an age matched non-collision sport control athlete cohort (Svaldi et al. 2017). In a clinical population, use of a controlled $\mathrm{CO}_{2}$ challenge paired with FMRI to assess CVR in a symptomatic adolescent population found globally decreased CVR in adolescent subjects with post-concussion syndrome, relative to healthy controls (Mutch et al. 2016). In a study using transcranial doppler, Becelewski and Pierzchala (2003) also reported reduced CVR in younger subjects ( $<30 \mathrm{yrs})$ following mTBI. While not directly measuring CVR, several studies have reported CBF reductions in sports-related mTBI populations, relative to healthy controls, both on an acute (Maugans et al. 2012; Wang et al. 2016) and relatively chronic (3-12 months postinjury) basis (Bartnik-Olson et al. 2014; Wang et al. 2016).

In contrast to prior literature, there was no evidence for a contribution of participant age to the prediction of CVR alterations as a function of accumulated HAEs. It is worth noting that previous studies that have revealed an agedependence were in cases of clinically-diagnosed mTBI in adults (Chan et al. 
2015; Mutch et al. 2014), wherein larger ranges of subject ages (here being limited to 14-18) may be associated with greater age-related variation in repair processes.

Existence of a minimum individual HAE threshold From this work, it may be hypothesized that (1) effects associated with accumulation of subconcussive HAEs below an existing magnitude threshold are effectively negated by natural repair processes and (2) magnitudes associated with the majority of HAEs experienced by athletes fall below this threshold. Though, the prediction of CVR changes from nCPTA measures was statistically significant over a wide range of minimum HAE thresholds (e.g., $35 \mathrm{~g} \leq T h \leq 70 \mathrm{~g}$; see Fig. 3a), all thresholds in this range are above the 50th percentile of impacts experienced by the soccer athletes, indicating that at least half of subconcussive events typically experienced by the cohort of athletes were not predictive of CVR changes from Pre. The observed optimal model relationship ( $T h=50 \mathrm{~g}$ ) corresponds to roughly the 75 th percentile of all HAEs experienced by the soccer athletes, and it is likely that significant fits at lower thresholds represent estimates made noisier by inclusion of less relevant subconcussive event data. This finer-grade analysis is expands on our report of $50 \mathrm{~g}$ serving as an optimal predictive threshold in high school football athletes (Bari et al. 2018), and provides a more practical day-to-day predictive tool than prior yearly exposure thresholds (e.g., such as preliminary thresholds derived from diffusion-weighted imaging) (Lipton et al. 2013).

The simple presence of such a minimum trauma-inducing threshold is, in part, supported by prior work suggesting that there is a minimum level of aggregate exposure to subconcussive events required for observation of increased risk of long-term neurological consequences (Montenigro et al. 2017). The extension of this basic hypothesis - that effects observed are likely from a minority of the total events experienced by an athlete-is also consistent with prior findings in adult amateur soccer athletes (Lipton et al. 2013; Stewart et al. 2017) and both collegiate (Slobounov et al. 2017) and high school (Abbas et al. 2015a) football athletes. Specifically, amateur soccer athletes in the highest quartile for frequency of heading exhibited significant associations between heading frequency and neurocognitive symptoms (Lipton et al. 2013; Stewart et al. 2017) and white matter microsctructural changes (Lipton et al. 2013); collegiate football athletes 
who experienced greater numbers of "high level" subconcussive HAEs (>80 g) per day were more likely to exhibit changes relative to pre-season in multiple neuroimaging measures (Slobounov et al. 2017); and high school football athletes exhibited greater changes in resting state functional connectivity during periods of elevated HAE exposure rates (Abbas et al. 2015a).

Competition between injury and repair CVR decreases exhibited throughout the season by the cohort studied here are hypothesized to be the beginning of the "neurometabolic energy crisis" (Giza and Hovda 2001), where a disruption in ionic signaling causes an imbalance between metabolic demand and CBF, that characterizes symptomatic mTBI and is thought to be caused by disruption of cerebrovascular coupling following mechanical insult. CVR dysregulation is likely a contributor to this disruption, as CVR is a crucial regulator of CBF and an important mechanism in neurovascular coupling (Ellis et al. 2016; Len and Neary 2011; Golding et al. 1999; Giza and Hovda 2001).

The later-season development (i.e., at $\operatorname{In} 2$ ) of a predictive relationship between cumulative subconcussive HAE exposure and the persistent CVR changes (e.g., Fig. 1) is hypothesized to reflect the outcome of a competition between neurovascular signaling disruption, brought about by the mechanical insult of HAEs, and natural repair processes working toward re-stabilization of ionic signaling pathways that mediate cerebrovascular coupling (Ellis et al. 2016). As each HAE occurs, some amount of insult correlated with event magnitude will accrue, initiating a repair process. For low levels of accumulated insult, this repair process should be a dominant factor, likely precluding the observation of a tight relationship between subconcussive HAEs and changes in CVR. However, as the cumulative level of insult grows, the ability of the body to repair this damage in an immediate timeframe would be expected to decrease, allowing the accumulation of subconcussive trauma to dominate. Predictability of CVR changes from cumulative HAE exposure in the latter half of the season would therefore be reflective of the rate of supra-threshold HAE accrual surpassing the rate of repair/adaptation, such that athletes experiencing greater subconcussive HAE accumulation exhibit progressively greater decreases in CVR. Finally, under this model, the diminished predictive coupling between CVR changes and HAE 
exposure at the Post session is well-explained by a return to the dominance of repair processes in the month of rest following cessation of insult accumulation.

\section{Implications for collision-sport athlete health Our findings of} cerebrovascular brain changes - consistent in direction with changes associated with diagnosed injury - in adolescent athletes indicates that lack of symptoms, even in the absence of acute history of injury, does not imply a truly healthy brain. This highlights the need for active monitoring of athlete exposure to HAEs, with the possibility that preseason (i.e., baseline) assessment of individual measures will also be of appreciable value in predicting the (near-term) health of an athlete. Critically, it is not only the counting of (subconcussive) HAEs that is of value, but also monitoring of the magnitude of HAEs typically experienced by the participant on a given day. While high normalized levels of exposure were not predictive of CVR changes early in the season, it is logical to infer that an individual for whom this high normalized exposure is sustained later into the season will eventually move into a region for which the predictive modeling developed herein will apply. As such, early season measures could effectively be used to identify athletes who are may experience undesirable levels of HAE accumulation, and for whom intervention (e.g., changes in training regimen, required rest, etc.) could prevent the later observation of changes in CVR or other measures of brain function and structure.

Limitations of current work Issues to be considered when interpreting the findings of this work include limitations associated with the relatively small sample size and concerns regarding the efficacy of the sensors worn to collect head acceleration telemetry. Regarding the sample size, larger studies of multiple teams spanning various age ranges and levels of competition will allow for exploration of more advanced longitudinal models and the inclusion of potentially relevant covariates such as history of concussion, years of exposure, and measures related to style of play. The current work supports the need for such a study to develop more generalizable, data-driven guidelines for prevention of mTBI. Regarding limitations surrounding the xPatch sensors, we note two primary considerations regarding the accuracy of the sensor as used in this study. First, the indisputable importance of HAEs that occur even in the absence of impacts to the head-e.g., 
whiplash from a car accident (Ommaya et al. 1968), or shaken baby syndrome (Duhaime et al. 1987) — dictates that analyses of acceleration events must not be limited to direct head impacts, recognizing that the confirmation of non-impact acceleration events presents a notable challenge. For this reason, the emphasis of this work is on assessment of the relationship of substantial (e.g., $\geq 20 \mathrm{~g}$ ) subconcussive HAEs, irrespective of direct physical contact, with cerebrovascular changes in the brain. We acknowledge that by always excluding recorded accelerations below $20 \mathrm{~g}$, we ignore approximately $25 \%$ of all recorded accelerations. However, given that the main finding of this paper is that including lower percentile (i.e., lower magnitude) events, when assessing the relationship between cumulative loading and CVR changes, substantively weakens the predictive relationship (see Fig. 3, left), there does not appear to be quantitative evidence that including accelerations below $20 \mathrm{~g}$ will advance the overall potential to detect or predict brain injury in the absence of neuroimaging. Second, the $30 \%$ RMS error range on individual acceleration events recorded using xPatch (Cummiskey et al. 2017) adds noise to the effort to identify a minimum threshold for HAEs. To this end, we have reported thresholds both as raw PTA values and as percentiles to emphasize that relative relationships of individual HAEs to population statistics are most relevant in this work.

\section{Conclusions}

Measures of daily subconcussive HAE accumulation over a season of play in female high school soccer athletes explained a significant percentage of the variance in cerebrovascular brain changes, consistent with symptomatic mTBI, observed in the athletes during the course of a competition season. This finding not only highlights the importance of daily monitoring of exposure to HAEs, but also illustrates the importance of collecting baseline (i.e., pre-exposure) measurements. Further, this work emphasizes the need for longitudinal studies across larger populations - participating at multiple levels of competition - to achieve generalizable characterizations of the relationship between HAE accumulation and biomarkers traditionally associated with mTBI that may permit early intervention to prevent similar changes in future athletes. 


\section{Acknowledgments}

This work was funded in part by support from the BrainScope Company, as part of a grant obtained from the GE-NFL Head Health Initiative, and by General Electric Healthcare.

\section{Funding}

This study was funded in part by the Indiana Clinical and Translational Sciences Institute Spinal Cord and Brain Injury Research Fund (Grant \#SCBI 207-32), and the BrainScope Company (as part of a grant from the GE-NFL Head Health Initiative).

Compliance with ethical standards

Conflict of interest The authors declare that they have no conflict of interest.

Ethical approval All procedures performed in studies involving human participants were in accordance with the ethical standards of the Purdue Institutional Review Board and with the 1964 Helsinki declaration and its later amendments or comparable ethical standards.

Informed consent Informed consent was obtained from all participants of 18 years and above, and parental consent and participant assent were obtained for all participants under the age of 18 .

\section{Electronic supplementary material}

\section{Fig. S1}

Distributions of estimated nCPTA coefficient $\left(\hat{x}_{1, T h, j}\right)$ at each follow up session. Distributions were computed by generating 10,000 bootstrapped randomly sampled observations and fitting the model for each sample. Distributions outlined in red delineate distributions that are significantly different from zero $(\alpha=0.05$, nonparametric $95 \%$ CI, 10,000 bootstraps) (EPS $12938 \mathrm{~kb}$ ) 


\section{Fig. S2}

Distributions of estimated $\widehat{R^{2}} T h, j$ value at each follow-up session. Distributions were computed by generating 10,000bootstrapped randomly sampled observations and fitting the model for each sample. Distributions outlined in red delineate distributions that are significantly different from zero $(\alpha=0.05$, non-parametric $95 \%$ CI, 10,000 bootstraps) (EPS $13264 \mathrm{~kb}$ )

\section{References}

Abbas, K., Shenk, T. E., Poole, V. N., Breedlove, E. L., Leverenz, L. J., Nauman, E. A., et al. (2015a). Alteration of default mode network in high school football athletes due to repetitive subconcussive mild traumatic brain injury: a resting-state functional magnetic resonance imaging study. Brain Connectivity, 5(2), 91-101. https://doi.org/10.1089/brain.2014.0279 .

Abbas, K., Shenk, T. E., Poole, V. N., Robinson, M. E., Leverenz, L. J., Nauman, E. A., et al. (2015b). Effects of repetitive subconcussive brain injury on the functional connectivity of Default Mode Network in high school football athletes. Developmental Neuropsychology, 40(1), 51-56.

https://doi.org/10.1080/87565641.2014.990455 .

Bahrami, N., Sharma, D., Rosenthal, S., Davenport, E. M., Urban, J. E., Wagner, B., et al. (2016). Subconcussive Head Impact Exposure and White Matter Tract Changes over a Single Season of Youth Football. Radiology, 281(3), 919-926. https://doi.org/10.1148/radiol.2016160564 .

Bailes, J. E., Petraglia, A. L., Omalu, B. I., Nauman, E., \& Talavage, T. (2013). Role of subconcussion in repetitive mild traumatic brain injury. Journal of Neurosurgery, 119(5), 1235-1245. https://doi.org/10.3171/2013.7.JNS121822 .

Bari, S., Svaldi, D. O., Jang, I., Shenk, T., Poole, V. N., Lee, T., et al. (2018). 
Dependence on subconcussive impacts of brain metabolism in collision sport athletes: an MR spectroscopic study. Brain Imaging and Behavior. https://doi.org/10.1007/s11682-018-9861-9 .

Barkhoudarian, G., Hovda, D. A., \& Giza, C. C. (2011). The molecular pathophysiology of concussive brain injury. Clinical Sports Medicine, 30(1), $33-48$.

Bartnik-Olson, B. L., Holshouser, B., Wang, H., Grube, M., Tong, K., Wong, V., et al. (2014). Impaired neurovascular unit function contributes to persistent symptoms after concussion: a pilot study. Journal of Neurotrauma, 31(17), 1497-1506. https://doi.org/10.1089/neu.2013.3213 .

Bazarian, J. J., Zhu, T., Zhong, J., Janigro, D., Rozen, E., Roberts, A., et al. (2014). Persistent, long-term cerebral white matter changes after sports-related repetitive head impacts. [Observational Study Research Support, Non-U.S. Gov't]. PLoS One, 9(4), e94734. https://doi.org/10.1371/journal.pone.0094734 .

Becelewski, J., \& Pierzchala, K. (2003). Cerebrovascular reactivity in patients with mild head injury. Neurologia i Neurochirurgia Polska, 37(2), 339-350.

Breedlove, E. L., Robinson, M., Talavage, T. M., Morigaki, K. E., Yoruk, U., O'Keefe, K., et al. (2012). Biomechanical correlates of symptomatic and asymptomatic neurophysiological impairment in high school football. [Research Support, Non-U.S. Gov't Research Support, U.S. Gov't, Non-P.H.S.]. Journal of Biomechanics, 45(7), 1265-1272. https://doi.org/10.1016/j.jbiomech.2012.01.034 .

Bright, M. G., \& Murphy, K. (2013). Reliable quantification of BOLD fMRI cerebrovascular reactivity despite poor breath-hold performance. Neuroimage, 83, 559-568. https://doi.org/10.1016/j.neuroimage.2013.07.007 .

Broshek, D. K., Kaushik, T., Freeman, J. R., Erlanger, D., Webbe, F., \& Barth, J. T. (2005). Sex differences in outcome following sports-related concussion. 
Journal of Neurosurgery, 102(5), 856-863.

Chan, S. T., Evans, K. C., Rosen, B. R., Song, T. Y., \& Kwong, K. K. (2015). A case study of magnetic resonance imaging of cerebrovascular reactivity: a powerful imaging marker for mild traumatic brain injury. Brain Injury, 29(3), 403-407. https://doi.org/10.3109/02699052.2014.974209 .

Chun, I. Y., Mao, X., Breedlove, E. L., Leverenz, L. J., Nauman, E. A., \& Talavage, T. M. (2015). DTI Detection of Longitudinal WM Abnormalities Due to Accumulated Head Impacts. [Research Support, Non-U.S. Gov't].

Developmental Neuropsychology, 40(2), 92-97.

https://doi.org/10.1080/87565641.2015.1020945 .

Cummiskey, B., Schiffmiller, D., Talavage, T. M., Leverenz, L., Meyer, J. J., Adams, D., et al. (2017). Reliability and accuracy of helmet-mounted and headmounted devices used to measure head accelerations. Proceedings of the Institution of Mechanical Engineers, Part P: Journal of Sports Engineering and Technology, 231(2), 144-153. https://doi.org/10.1177/1754337116658395.

Davenport, E. M., Apkarian, K., Whitlow, C. T., Urban, J. E., Jensen, J. H., Szuch, E., et al. (2016). Abnormalities in Diffusional Kurtosis Metrics Related to Head Impact Exposure in a Season of High School Varsity Football. Journal of Neurotrauma, 33(23), 2133-2146. https://doi.org/10.1089/neu.2015.4267 .

Davenport, E. M., Whitlow, C. T., Urban, J. E., Espeland, M. A., Jung, Y., Rosenbaum, D. A., et al. (2014). Abnormal white matter integrity related to head impact exposure in a season of high school varsity football. [Research Support, N.I.H., Extramural]. Journal of Neurotrauma, 31(19), 1617-1624. https://doi.org/10.1089/neu.2013.3233 .

Delaney, J. S., Lacroix, V. J., Leclerc, S., \& Jonston, K. (2002). Concussions Among University Football and Soccer Players. Clinical Journal of Sport Medicine, 12, 331-338. 
Duhaime, A. C., Gennarelli, T. A., Thibault, L. E., Bruce, D. A., Margulies, S. S., \& Wiser, R. (1987). The shaken baby syndrome. A clinical, pathological, and biomechanical study. Journal of Neurosurgery, 66(3), 409-415. https://doi.org/10.3171/jns.1987.66.3.0409 .

Ellis, M. J., Ryner, L. N., Sobczyk, O., Fierstra, J., Mikulis, D. J., Fisher, J. A., et al. (2016). Neuroimaging Assessment of Cerebrovascular Reactivity in Concussion: Current Concepts, Methodological Considerations, and Review of the Literature. Frontiers in Neurology, 7, 61. https://doi.org/10.3389/fneur.2016.00061 .

Giza, C. C., \& Hovda, D. A. (2001). The Neurometabolic Cascade of Concussion. Journal of Athletic Training, 36(3), 228-235.

Golding, E. M., Robertson, C. S., \& Bryan Jr., R. M. (1999). The consequences of traumatic brain injury on cerebral blood flow and autoregulation: a review. Clinical and Experimental Hypertension, 21(4), 299-332.

Johnson, B., Neuberger, T., Gay, M., Hallett, M., \& Slobounov, S. (2014). Effects of subconcussive head trauma on the default mode network of the brain. [Research Support, Non-U.S. Gov't]. Journal of Neurotrauma, 31(23), 19071913. https://doi.org/10.1089/neu.2014.3415 .

Kastrup, A., Gunnar, K., Neumann-Haefelin, T., \& Moseley, M. (2001). Assessment of cerebrovascular reactivity with functional magnetic resonance imaging: comparison of $\mathrm{C} 02$ and Breath Holding. Magnetic Resonance Imaging, 19, 13-20.

Koerte, I. K., Lin, A. P., Willems, A., Muehlmann, M., Hufschmidt, J., Coleman, M. J., et al. (2015). A review of neuroimaging findings in repetitive brain trauma. Brain Pathology, 25(3), 318-349.

https://doi.org/10.1111/bpa.12249 .

Len, T. K., \& Neary, J. P. (2011). Cerebrovascular pathophysiology following 
mild traumatic brain injury. Clinical Physiology and Functional Imaging, 31(2), 85-93. https://doi.org/10.1111/j.1475-097X.2010.00990.x .

Len, T. K., Neary, J. P., Asmundson, G. J., Candow, D. G., Goodman, D. G., Bjornson, B., et al. (2013). Serial monitoring of CO2 reactivity following sport concussion using hypocapnia and hypercapnia. Brain Injury, 27(3), 346-353. https://doi.org/10.3109/02699052.2012.743185 .

Lewis, P. M., Czosnyka, M., Smielewski, P., \& Pickard, J. D. (2014). Cerebrovascular Autoregulation and Monitoring of Cerebrovascular Reactivity. In E. H. Lo, M. Ning, J. Lok, \& M. J. Whalen (Eds.), Vascular Mechanisms in CNS and Trauma. New York: Springer.

Lipp, I., Murphy, K., Caseras, X., \& Wise, R. G. (2015). Agreement and repeatability of vascular reactivity estimates based on a breath-hold task and a resting state scan. Neuroimage, 113, 387-396. https://doi.org/10.1016/j.neuroimage.2015.03.004 .

Lipton, M. L., Kim, N., Zimmerman, M. E., Kim, M., Stewart, W. F., Branch, C. A., et al. (2013). Soccer Heading is Assciated with White Matter Microstructural and Cognitive Abnormalities. Radiology, 268(3), 850-857.

Marar, M., McIlvain, N. M., Fields, S. K., \& Comstock, R. D. (2012). Epidemiology of concussions among United States high school athletes in 20 sports. The American Journal of Sports Medicine, 40(4), 747-755. https://doi.org/10.1177/0363546511435626.

Maugans, T. A., Farley, C., Altaye, M., Leach, J., \& Cecil, K. M. (2012). Pediatric sports-related concussion produces cerebral blood flow alterations. Pediatrics, 129(1), 28-37. https://doi.org/10.1542/peds.2011-2083 .

McAllister, T. W., Ford, J. C., Flashman, L. A., Maerlender, A., Greenwald, R. M., Beckwith, J. G., et al. (2014). Effect of head impacts on diffusivity measures in a cohort of collegiate contact sport athletes. Neurology, 82(1), 63- 
69. https://doi.org/10.1212/01.wnl.0000438220.16190.42 .

McCuen, E. C., Svaldi, D. O., Breedlove Morigaki, K., Kraz, N., Cummiskey, B., Breedlove, E., et al. (2015). Colleigate Women's Soccer Players Suffer Greater Cumulative Head Impacts than their High School Counterparts. Journal of Biomechanics, 48(13), 3720-3723.

AQ3

Meaney, D. F., \& Smith, D. H. (2011). Biomechanics of concussion. Clinics in Sports Medicine, 30(1), 19-31, vii. https://doi.org/10.1016/j.csm.2010.08.009 .

Montenigro, P. H., Alosco, M. L., Martin, B. M., Daneshvar, D. H., Mez, J., Chaisson, C. E., et al. (2017). Cumulative Head Impact Exposure Predicts Later-Life Depression, Apathy, Executive Dysfunction, and Cognitive Impairment in Former High School and College Football Players. Journal of Neurotrauma, 34(2), 328-340. https://doi.org/10.1089/neu.2016.4413 .

Morris, B. (2015). Why Is the U.S. So Good at Women's Soccer? http://fivethirtyeight.com/datalab/why-is-the-u-s-so-good-at-womens-soccer/ .

Mutch, W. A., Ellis, M. J., Graham, M. R., Wourms, V., Raban, R., Fisher, J. A., et al. (2014). Brain MRI CO2 stress testing: a pilot study in patients with concussion. PLoS One, 9(7), e102181.

https://doi.org/10.1371/journal.pone.0102181.

Mutch, W. A., Ellis, M. J., Ryner, L. N., Ruth Graham, M., Dufault, B., Gregson, B., et al. (2016). Brain magnetic resonance imaging CO2 stress testing in adolescent postconcussion syndrome. Journal of Neurosurgery, 125(3), 648-660. https://doi.org/10.3171/2015.6.JNS15972 .

Ommaya, A. K., Faas, F., \& Yarnell, P. (1968). Whiplash injury and brain damage: an experimental study. JAMA, 204(4), 285-289.

Peirce, J. W. (2007). PsychoPy--Psychophysics software in Python. Journal of 
Neuroscience Methods, 162(1-2), 8-13.

https://doi.org/10.1016/j.jneumeth.2006.11.017 .

Poole, V. N., Abbas, K., Shenk, T. E., Breedlove, E. L., Breedlove, K. M., Robinson, M. E., et al. (2014). MR spectroscopic evidence of brain injury in the non-diagnosed collision sport athlete. Developmental Neuropsychology, 39(6), 459-473. https://doi.org/10.1080/87565641.2014.940619.

Poole, V. N., Breedlove, E. L., Shenk, T. E., Abbas, K., Robinson, M. E., Leverenz, L. J., et al. (2015). Subconcussive hit characteristics predict deviant brain metabolism in football athletes. Developmental Neuropsychology, 40(1), 12-17. https://doi.org/10.1080/87565641.2014.984810 .

Robinson, M. E., Shenk, T. E., Breedlove, E. L., Leverenz, L. J., Nauman, E. A., \& Talavage, T. M. (2015). The role of location of subconcussive head impacts in FMRI brain activation change. Developmental Neuropsychology, 40(2), 74-79. https://doi.org/10.1080/87565641.2015.1012204 .

Schultz, V., Stern, R. A., Tripodis, Y., Stamm, J. M., Wrobel, P., Lepage, C., et al. (2017). Age at First Exposure to Repetitive Head Impacts Is Associated with Smaller Thalamic Volumes in Former Professional American Football Players. Journal of Neurotrauma. https://doi.org/10.1089/neu.2017.5145 .

Shenk, T. E., Robinson, M. E., Svaldi, D. O., Abbas, K., Breedlove, K. M., Leverenz, L. J., et al. (2015). FMRI of visual working memory in high school football players. Developmental Neuropsychology, 40(2), 63-68. https://doi.org/10.1080/87565641.2015.1014088 .

Slobounov, S. M., Walter, A., Breiter, H. C., Zhu, D. C., Bai, X., Bream, T., et al. (2017). The effect of repetitive subconcussive collisions on brain integrity in collegiate football players over a single football season: A multi-modal neuroimaging study. Neuroimage Clin, 14, 708-718. https://doi.org/10.1016/j.nicl.2017.03.006 . 
Stamm, J. M., Koerte, I. K., Muehlmann, M., Pasternak, O., Bourlas, A. P., Baugh, C. M., et al. (2015). Age at First Exposure to Football is Associated with Altered Corpus Callosum White Matter Microstructure in Former Professional Football Players. Journal of Neurotrauma. https://doi.org/10.1089/neu.2014.3822 .

Stein, T. D., Alvarez, V. E., \& McKee, A. C. (2015). Concussion in Chronic Traumatic Encephalopathy. Current Pain and Headache Reports, 19(10), 47. https://doi.org/10.1007/s11916-015-0522-z .

Stewart, W. F., Kim, N., Ifrah, C., Bachrach, T. A., Zimmerman, M. E., Kim, M., et al. (2017). Symptoms from repeated intentional and unintentional head impact in soccer players. Neurology, 88, 901-908.

Svaldi, D. O., Joshi, C., Robinson, M. E., Shenk, T. E., Abbas, K., Nauman, E. A., et al. (2015). Cerebrovascular reactivity alterations in asymptomatic high school football players. Developmental Neuropsychology, 40(2), 80-84. https://doi.org/10.1080/87565641.2014.973959 .

Svaldi, D. O., McCuen, E. C., Joshi, C., Robinson, M. E., Nho, Y., Hannemann, R., et al. (2017). Cerebrovascular reactivity changes in asymptomatic female athletes attributable to high school soccer participation. Brain Imaging and Behavior, 11(1), 98-112. https://doi.org/10.1007/s11682-016-9509-6 .

Tagge, C. A., Fisher, A. M., Minaeva, O. V., Gaudreau-Balderrama, A., Moncaster, J. A., Zhang, X.-L., et al. (2018). Concussion, microvascular injury, and early tauopathy in young athletes after impact head injury and an impact concussion mouse model. Brain, awx350-awx350.

https://doi.org/10.1093/brain/awx350 .

Talavage, T. M., Nauman, E. A., Breedlove, E. L., Yoruk, U., Dye, A. E., Morigaki, K. E., et al. (2014). Functionally-detected cognitive impairment in high school football players without clinically-diagnosed concussion. Journal of Neurotrauma, 31(4), 327-338. https://doi.org/10.1089/neu.2010.1512 . 
Talavage, T. M., Nauman, E. A., \& Leverenz, L. J. (2015). The Role of Medical Imaging in the Recharacterization of Mild Traumatic Brain Injury Using Youth Sports as a Laboratory. [Review]. Frontiers in Neurology, 6, 273. https://doi.org/10.3389/fneur.2015.00273 .

Wang, Y., Nelson, L. D., LaRoche, A. A., Pfaller, A. Y., Nencka, A. S., Koch, K. M., et al. (2016). Cerebral Blood Flow Alterations in Acute Sport-Related Concussion. Journal of Neurotrauma, 33(13), 1227-1236. https://doi.org/10.1089/neu.2015.4072 . 